\title{
Clinical Features of Partial Atrophic Tongue Associated with Candida
}

\author{
Terai $\mathrm{H}^{1 *}$, Fukui $\mathrm{N}^{2}$, Kasuya $\mathrm{S}^{2}$, Hashiguchi $\mathrm{N}^{3}$, Ueno $\mathrm{T}^{4}$ \\ ${ }^{1}$ Associate Professor, Department of Dentistry and Oral Surgery, Osaka Medical College, Daigaku-machi, Takatuki-shi, Osaka, Japan. \\ ${ }^{2}$ Fellow, Department of Dentistry and Oral Surgery, Osaka Medical College, Daigaku-machi, Takatuki-shi, Osaka, Japan. \\ ${ }^{3}$ Assistant Professor, Department of Dentistry and Oral Surgery, Osaka Medical College, Daigaku-machi, Takatuki-shi, Osaka, Japan. \\ ${ }^{4}$ Professor, Department of Dentistry and Oral Surgery, Osaka Medical College, Daigaku-machi, Takatuki-shi, Osaka, Japan.
}

Research Article

\section{Abstract}

Background: Clinical features of partial atrophic tongue associated with Candidia including median rhomboid glossitis have not been established.

Methods: We enrolled 90 patients with atrophic tongue who were referred to our department with the chief symptom of tongue pain when eating spicy or hot food. They were diagnosed as having oral candidiasis, and were successfully treated with anti-fungal agents. The patients were divided into groups, based on partial atrophic $(n=29)$ or total atrophic tongue $(n$ = 61). Age at presentation, sex, duration of tongue pain, other signs and symptoms, complete medical history, prior treatment for the symptom, and a culture test were obtained for each patient.

Results: Statistically significant differences between the groups were observed for age distribution, predisposing factors for candidiasis, and culture tests.

Conclusion: Patients with partial atrophic tongue were younger, were generally healthier, and Candida species were less frequency isolated in the culture than the patients with total atrophic tongue.

Keywords: Oral Candidiasis; Partial Atrophic Tongue; Clinical Features of Candidiasis.

\section{Introduction}

Candida infections are the most frequently encountered fungal infections of the oral mucosa. The manifestations of oral candidiasis vary, and numerous forms have been noted, including atrophic tongue or erythematous candidiasis of the tongue $[1,2]$. Median rhomboid glossitis has traditionally been regarded as a developmental anomaly resulting in the failure of the tuberculum impar to withdraw during tongue embryogenesis. However, many recent studies have also implicated Candida albicans as a probable cause; thus, median rhomboid glossitis, which is a partial atrophic pattern of the tongue, is now classified as a Candida-associated lesion [2-4]. In addition, symptoms other than median rhomboid glossitis can occur with Candida-associated partial atrophic tongue (PAT), as we have reported previously [5]. Etiologic factors of PAT including median rhomboid glossitis have not been established. At first sight, the clinical findings of PAT resemble those of geographic tongue. However, PAT not only had partial atrophic change of the tongue surface but also tongue pain on eating spicy or hot food, and it was incurable without anti-fungal treat- ment. On the other hand, geographic tongue is usually asymptomatic, and can regress over a period of time ranging from a few hours to several weeks [4]. Therefore, we believe that functional pain on eating should be considered one of important signs of inflammation or infection [5]. Samaranayake et al reported that erythematous candidiasis may arise as a consequence of persistent acute pseudomembranous candidiasis when pseudomembranes are shed, may develop de novo, or may precede pseudomembranous candidiasis [2].

In this study, we compared the clinical features of PAT and total atrophic tongue (TAT) to identify principal factors associated with PAT.

\section{Materials and Methods}

We enrolled 90 patients with atrophic tongue between January 2003 and December 2012 who were referred to our department with the chief symptom of tongue pain when eating spicy or hot food. The diagnosis of atrophic candidiasis was confirmed by

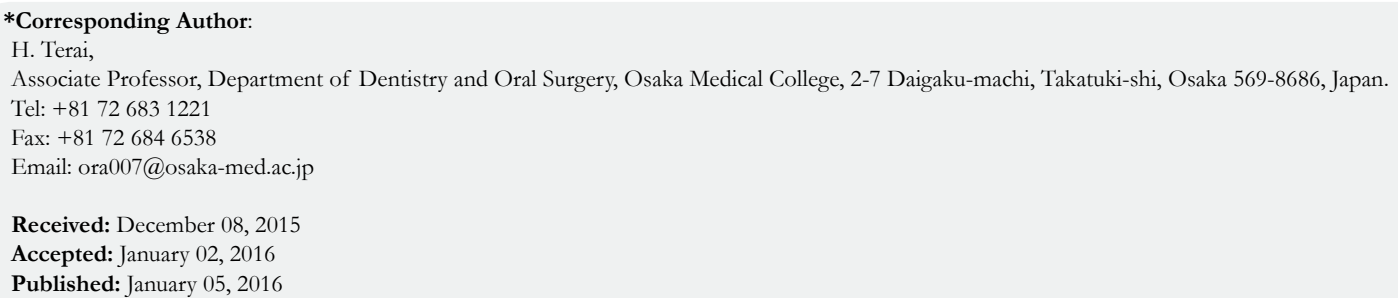

Copyright: Terai $\mathbf{H}^{\circ}$ 2016. This is an open-access article distributed under the terms of the Creative Commons Attribution License, which permits unrestricted use, distribution and reproduction in any medium, provided the original author and source are credited. 
a favorable response to anti-fungal treatment; improvement of tongue pain and regeneration of filiform papilla of the tongue were obtained in all patients within 2 to 4 weeks of anti-fungal treatment. Patients who did not experience tongue pain when eating were excluded as geographic tongue or others. For each patient, age at presentation, sex, symptom (tongue pain) duration, other signs and symptoms, complete medical history, and prior treatment or medications for the symptom were obtained.

Retrospectively, the 90 patients were divided into groups based on the presence of PAT $(n=29)$ or TAT $(n=61)$. Patients with PAT had clear atrophic margins on the tongue dorsum (Figure $1,2)$, while patients with TAT had atrophy over $80 \%$ of the dorsum. Two patients with median rhomboid glossitis were included in PAT. Patients with unclear atrophic tongue margins were excluded.

For culture tests, samples from the atrophic site in both groups were collected using the scratch smear method and incubated on Sabouraud agar medium at $35^{\circ} \mathrm{C}$ for 3 days. All of the patients, regardless of culture test results, were successfully treated with an antifungal agent (25-mg miconazole gel administered four times daily for 2 to 4 weeks).

Mann-Whitney $U$ and Fisher exact tests were used to investigate differences in clinical features between the two groups. Statistical significance was defined as $p<0.05$.

This study was approved by the local committee.

\section{Results}

The results are shown in Table 1 . The mean age was significantly different between the groups (PAT, $59.0 \pm 13.6$ years; TAT, 69.9 \pm 8.0 years, $p=0.0036$ ). The distributions of sex and disease duration were similar between the groups, with approximately $70 \%$ of the patients being women and an approximate 4-month dis- ease duration in both groups. Prior medications for tongue pain administered at other clinics were topical steroids (PAT: 5, TAT: 16) and gargled povidone iodine (PAT: 2, TAT: 3), which failed to resolve their symptoms. The medical history revealed the following predisposing factors for candidiasis (PAT: $20.7 \%$, TAT: 60.7\%): diabetes mellitus, systemic steroids, antibiotic therapy, anemia, and anti-cancer drugs. In the culture test, Candida species were isolated in 17 (58.6\%) patients in the PAT group and 53 patients $(86.9 \%)$ in the TAT group. Almost all of the species were Candida albicans; Candida glabrata was detected in 1 PAT patient, and Candida tropicalis was detected in 1 TAT patient. False negative results were obtained in residual 20 patients (PAT:12, TAT: 8).

There were significant differences in age distributions $(p=0.0036)$, predisposing factors for candidiasis $(p=0.0003)$ and positive rate of Candida species $(p=0.00037)$ between two groups. In other words, these results showed that patients with PAT were younger, were in better general condition, and Candida species were less frequently isolated than the patients with TAT.

\section{Discussion}

Pseudomembranous candidiasis is easily diagnosed. However, erythematous candidiasis (atrophic tongue) may be difficult to diagnose clinically, because pseudohyphae in this candidiasis are relatively few $[6,7]$. Therefore, the diagnosis of oral candidiasis is generally based on clinical signs and symptoms in conjunction with a thorough medical history [7]. One fifth false-negative results were obtained by the culture test in this study, which may be support above mentioned idea.

The transition of Candida species from a harmless commensal to a pathogenic microorganism is complex and involves host and candidal factors. The development of oral candidiasis is thus the result of an imbalance between fungal virulence factors and host defences [6]. However, candida-associated atrophic tongue, usually presenting with a total atrophic pattern, is well known, but its

Figure 1. Case 1 of partial atrophic tongue. (a) Atrophic changes with clear margins were visible at the tongue dorsum. (b) Regeneration of filiform papillae is seen after 2 weeks of anti-fungal treatment.
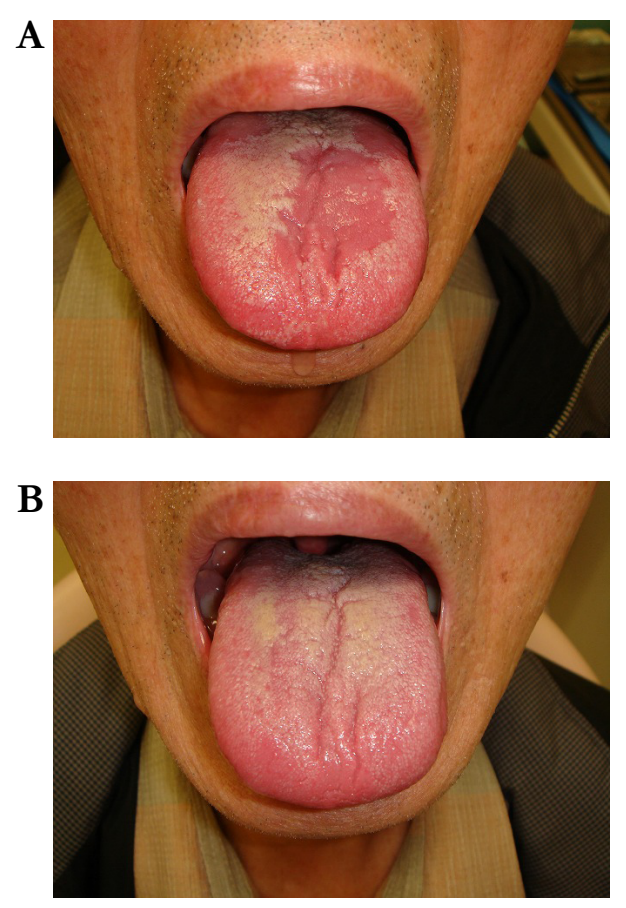
Figure 2. Case 2 of partial atrophic tongue. (a). Atrophic changes were visible at the tongue dorsum. (b). Direct examination of the smear sample from the atrophic site showed some pseudohyphae of Candida albicans (Cyto Quick staining system, $\times 400)$. (c). Complete response was seen 2 weeks after anti-fungal treatment.

A

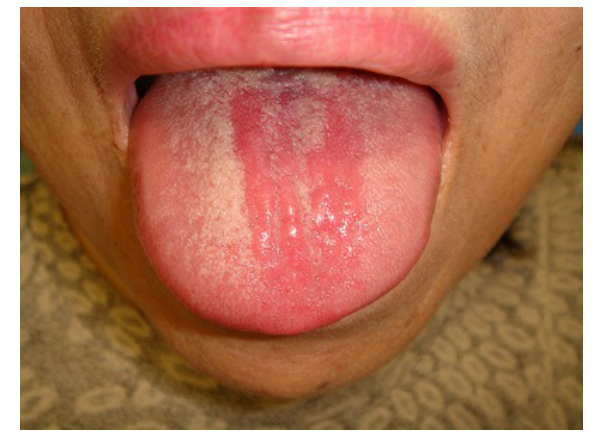

B

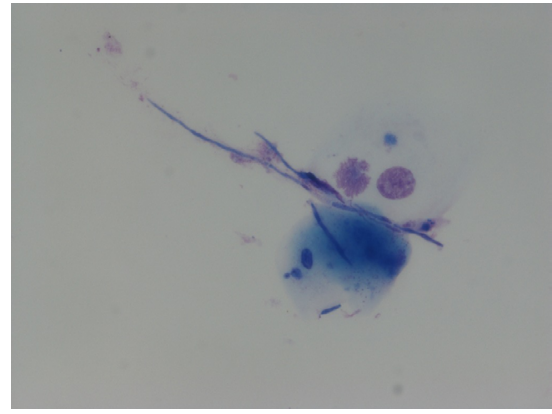

C

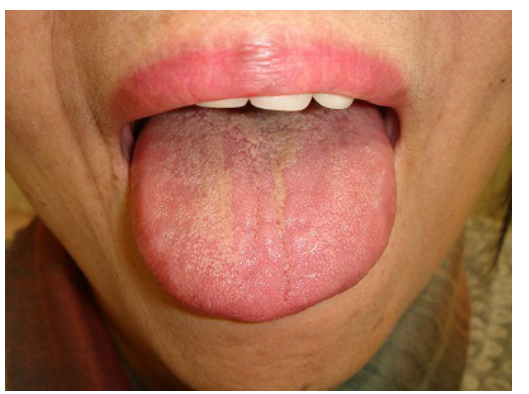

Table 1. Results

\begin{tabular}{|c|c|c|}
\hline & PAT $(\mathbf{n}=\mathbf{2 9})$ & TAT $(\mathbf{n}=\mathbf{6 1})$ \\
\hline Age $^{\uparrow}$ & $\begin{array}{c}\text { mean age: } 59.0 \pm 13.6 \text { years range: } \\
27 \sim 82 \text { years old }\end{array}$ & $\begin{array}{c}\text { mean age: } 69.9 \pm 8.0 \text { years range: } \\
44 \sim 82 \text { years old }\end{array}$ \\
\hline Sex & 7 men, 22 women & 16 men, 45 women \\
\hline Disease duration (Months) & $4.8 \pm 4.5$ & $4.4 \pm 3.2$ \\
\hline Prior medications & $+7:-22$ & $+19:-42$ \\
\hline Predisposing factors for candidiasis* & $+6:-23$ & $+37:-24$ \\
\hline Culture test ${ }^{*}$ (Candida spp.) & $+17:-12$ & $+53:-8$ \\
\hline
\end{tabular}

PAT: partial atrophic tongue, TAT: total atrophic tongue.

${ }^{\uparrow} P<0.01$, Mann-Whitney $\mathrm{U}$ test, ${ }^{*} P<0.01$, Fisher's exact test

pathogenesis is not elucidated fully. Kimori et al [8] reported that high Candida colony-forming units, low stimulated saliva flow rate and advanced age were identified as closely associated factors for the risk of development of atrophic tongue. Although, the results of this study include advanced age in the patients with atrophic tongue, patients with PAT were younger than patients with TAT. Then, a question arises that whether Candida-associated partial atrophy is one stage in the clinical course of total atrophy or not. We have not observed a transition between PAT and TAT in our clinical experience. The etiology of PAT may somewhat differ from that of TAT, and PAT may be established by more insignificant cause such as fatigue, stress and so on. Although it is the study in small groups, the results obtained in this study, including findings that the patients with PAT were younger and generally healthier than the patients with TAT might supportthis idea. Candidal virulence factors include the ability of Candida species to adhere to host surfaces and form biofilms, to resist host immune defence mechanisms and to release hydrolytic enzymes that can induce damage to host cell [9].

Further investigation is necessary to clarify the differences between these atrophic patterns for understanding of pathogenesis of oral candidiasis. 


\section{Conclusion}

Patients with partial atrophic tongue were younger, were generally healthier, and Candida species were less frequently isolated than the patients with total atrophic tongue.

\section{References}

[1]. Lynch DP (1994) Oral candidiasis. History, classification, and clinical presentation. Oral Surg Oral Med Oral Pathol 78(2): 189-193.

[2]. Samaranayake LP, Cheung LK, Samaranayake YH (2002) Candidiasis and other fungal disease of the mouth. Dermatol Ther 15(3): 251-269.

[3]. Wright BA, Fenwick F (1981) Candidiasis and atrophic tongue lesions. Oral
Surg Oral Med Oral Pathol 51(1): 55-61.

[4]. Rogers RS, Bruce AJ (2004) The tongue in clinical diagnosis. J Eur Acad Dermatol Venereol 18(3): 254-259.

[5]. Terai H, Shimahara M (2007) Partial atrophic tongue other than median rhomboid glossitis. Clin Exp Dermatol 32(4): 381-384.

[6]. Manfredi M, Polonelli L, Aguirre-Urizar JM, Carrozzo M, McCullough MJ (2013) Urban legends series: oral candidosis. Oral Dis 19(3): 245-261.

[7]. Farah CS, Lynch N, McCullough MJ (2010) Oral fungal infections: an update for the general practitioner. Aust Dent J 55(Suppl 1): 48-54.

[8]. Kimori H, Yamamoto K, Yamachika S, Tsurumoto A, Kamikawa Y, et al. (2015) Factors associated with the presence of atrophic tongue in patients with dry mouth. Gerodontology 32(1): 13-17.

[9]. Lim CS, Rosli R, Seow HF, Chong PP (2012) Candida and invasive candidiasis: back to basics. Eur J Clin Microbiol Infect Dis 31(1): 21-31. 